\title{
Influence of temporal resolution on the performance of Global Ionospheric Maps
}

\author{
Qi Liu · Manuel Hernández-Pajares · \\ Haixia Lyu • Andreas Goss
}

Received: date / Accepted: date

\begin{abstract}
Global ionosphere maps (GIM) computed from dual-frequency GNSS measurements have been widely used for monitoring ionosphere as well as providing ionospheric corrections in Space Geodesy since 1998. This work focuses on a comprehensive study of the influence of time resolution on GIM performance. One and a half solar cycle of the IGS GIM with higher time resolution and accuracy (the UPC-IonSAT Quarter-of-an-hour time resolution Rapid GIM, AKA uqrg) has been taken as baseline to downsample them to all possible sub-daily temporal resolutions. The performance of the resulting GIMs has been assessed by directly comparing with external Vertical Total Electron Content (VTEC) measurements from Jason altimeters over oceanic regions. In order to perform a complete assessment and analysis of involved GIMs, the influence of geographical position, solar and geomagnetic activity were also taken into account during more than one solar cycle. In addition, to have a clear view at the smaller time resolutions, a more accurate assessment, the dSTEC test based on external GNSS measurements not used in the GIM generation, was also done during two solstice and two equinox days in 2015 over continental regions. The assessment shows that discrepancy among GIMs with different time resolutions becomes more apparent at low latitudes and also at high solar-geomagnetic activity. The results also suggest that the accuracy for GIMs with time resolution smaller or equal to 60 minutes is consistent
\end{abstract}

\footnotetext{
Qi Liu

Universitat Politècnica de Catalunya (UPC-IonSAT), Barcelona, Spain

E-mail: q.liu@upc.edu

Manuel Hernández-Pajares, Haixia Lyu

Universitat Politècnica de Catalunya (UPC-IonSAT), Barcelona, Spain

IEEC, Barcelona, Spain

E-mail: manuel.hernandez@upc.edu

Andreas Goss

Deutsches Geodtisches Forschungsinstitut der Technischen Universitt Mnchen, Deutsches Geodtisches ForschungsinstitArcisstrae 21, 80333 Munich, Germany
} 
during the period from 2002 to 2019 and is more accurate than other GIMs with lower temporal resolution. Accordingly, high time resolution (including $15,30,45$ and 60 minutes) is recommended for the application of GIMs with the highest accuracy.

Keywords Global Navigation Satellite Systems · Ionosphere · Global Ionospheric Map - Temporal Resolution · Vertical Total Electron Content · Jason

\section{Introduction}

The ionosphere is a part of upper atmosphere of Earth (from approximately 60 to $1000 \mathrm{~km}$ above sea level) where molecules are ionized by high energy radiation and resultant free electrons significantly affect radio wave propagation([18,6]). For users of Global Navigation Satellite Systems (GNSS), the integral value of electron density along the satellite-to-receiver path, i.e. slant total electron content (STEC), causes a time delay and phase advance in pseudorange and carrier phase observations, respectively. Such ionospheric delay values range from meters to tens of meters (and up to few hundreds of meters in extreme cases), thus affecting the accuracy of single-frequency GNSS positioning $([17,23,2,25])$, but also being an important information affecting the convergence time in multi-frequency accurate GNSS positioning in realtime (see for instance [10]). The International GNSS Service (IGS) Working Group on Ionosphere has provided reliable GIM products since 1998, giving valuable information for ionospheric sounding and correction in space physics and satellite navigation fields, respectively $([4,11,27,15,13,22])$.

Currently, there are seven IGS ionosphere associated analysis centers (IAACs): Center for Orbit Determination in Europe (CODE); European Space Agency/ European Space Operations Center (ESA/ESOC); Jet Propulsion Laboratory (JPL); Universitat Politcnica de Catalunya (UPC); Chinese Academy of Sciences (CAS); Canadian Geodetic Survey of Natural Resources Canada (NRCan); Wuhan University (WHU). These IAACs, by means of different algorithms, produce GIMs in IONosphere Map EXchange(IONEX) format, being further combined in terms of common IGS ionospheric GIMs (a summary of algorithms for different IAACs can be seen in [22]). The temporal resolution of different IAAC-GIMs ranges from 2 hour (adopted among others by IGS, ESA/ESOC, JPL, UPC, WHU), 1 hour (e.g. CODE, NRcan, UPC), 30 minutes (CAS) and 15 minutes (UPC). It should be noted that UPC generates in particular two types of GIMs: upcg and uqrg, at final (few days) and rapid (one day) latencies, respectively. These two GIMs are based on the regional VTEC modelling from the TOMographic IOnosphere (TOMION-v1) model software. While upcg uses spline functions to interpolate regional VTEC for the generation of global TEC map with 2-hour resolution, uqrg adopts kriging interpolation with 15-minute resolution instead (see [7,8,21]. Since VTEC values are provided in fixed grid points, with spatial resolution of 2.5 degrees in latitude and 5 degrees in longitude every 15 minutes in GIMs, a bivariate 
interpolation in longitude and latitude and a linear time interpolation are used for the calculation of VTEC at arbitrary position and time (see [24]). In this context, the time dependence of ionosphere VTEC at each given sun-fixed (local-time and latitude) point is considered as linear.

The electron density of ionosphere can be highly variable due to many factors such as solar radiation, as well as plasma transport. In addition, the irregular variation of electron density under different space conditions, such as solar storms, geomagnetic storms, or traveling ionospheric disturbance (TID), will present a challenge for the modeling and interpolation of ionosphere $([16,9]$. Thus, the assumption that TEC varies linearly between consecutive VTEC maps during periods of 2 hours in a sun-fixed reference frame ([24]), might not be appropriate for the time interpolation of GIM. In the 2012 IGS workshop, a recommendation of higher temporal and spatial resolution (15 minutes and $1^{\circ} \times 1^{\circ}$, respectively) for the IGS combined GIM was proposed [3]. However, there are few studies about which GIM time resolution is most suitable for its application. Preliminary studies of different GIM temporal resolutions, based on spherical harmonic model and multi-scale representation as well as products from B-spline function, were performed by using GNSS data of one month $([26,5])$. It was found that 30 -minutes or 10 -minute GIM can obtain a noticeable improvement of the GIM accuracy compared with 2-hour GIM. A long period study was performed in [22] by comparing GIMs with 15 minutes, 1 hour and 2 hours time interval with data during one solar cycle. However, a comprehensive comparison of the performance of different temporal resolutions for the application of GIM was still pending.

VTEC derived from altimeter data have been widely utilized for the evaluation of GIM, because it is an independent and direct electron content observation (see for instance $[21,15,13]$ ). Therefore, we carried out a complete research of all possible temporal resolution for GIM and also studied the performance of involved GIMs compared with Jason altimeter data at different latitudes as well as various solar and geomagnetic activities from 2002 to 2019 (one and a half solar cycle) over the oceans. Another complementary assessment, the dSTEC assessment from GPS observations (equivalent but more accurate because it is based on carrier phase data and it is free from instrumental biases, see for instance [13]) was conducted by 59 GNSS receivers during two solstice and two equinox days in 2015 over continents. The external GPS receivers used for the dSTEC test is closer than the altimeter observations (above the oceans) to the GPS receivers used to compute the GIMs; and the accuracy of dSTEC reference observations is higher (better than 0.1 TECU) than the precision of the altimeter VTEC measurements (better than 1 TECU).

Section 2 will describe the input data for assessment in detail. Section 3 and section 4 briefly introduced the GIM assessment method of Jason-altimeter data and dSTEC-GPS observation, respectively. With the results from assessment, we thoroughly discussed the the performance of involved GIMs at complex space environment as well as different latitudes in section 5, with an overall conclusion included in section 6 . 


\section{Input Data}

In order to compare GIMs of different temporal resolutions in the best conditions, the most accurate GIM in IGS was selected [22]. This rapid GIM (uqrg), feed with dual-frequency carrier phase data only, is computed by combining (see [8] and [21]):

1. Ionospheric tomography (for estimating the carrier phase bias and electron densities with a Kalman filter updated every 30 seconds), and,

2. Kriging interpolation technique, performed every 15 minutes with a spatial resolution of 5 and 2.5 degree in longitude and latitude respectively.

The main input data were composed of twelve different GIMs derived from 15 minutes uqrg GIM, in order to evaluate the influence of temporal resolution on GIM application performance. The involved GIMs were obtained by direct downsampling of the daily uqrg GIM to all the multiples of 15 minutes within a day. The derived GIMs were named 'uxxg', being 'xx' the integer multiple. As it can be seen in Table 1, the names of twelve GIMs are listed in the first column with corresponding temporal resolution in the second column, and the common multiples for each GIM are indicated in the third column.

Table 1 The analyzed GIM temporal resolutions, derived as 24-hour divisor time intervals and multiple of the 15-minutes time resolution of the baseline GIM uqrg.

\begin{tabular}{ccc}
\hline GIM Id. & temporal resolution & common multiple \\
\hline uqrg & 15 minutes & $2^{0} \cdot 3^{0}=01$ \\
u02g & 30 minutes & $2^{1} \cdot 3^{0}=02$ \\
u03g & 45 minutes & $2^{0} \cdot 3^{1}=03$ \\
u04g & 1 hour & $2^{2} \cdot 3^{0}=04$ \\
u06g & 1 hour 30 minutes & $2^{1} \cdot 3^{1}=06$ \\
u08g & 2 hours & $2^{3} \cdot 3^{0}=08$ \\
u12g & 3 hours & $2^{2} \cdot 3^{1}=12$ \\
u16g & 4 hours & $2^{4} \cdot 3^{0}=16$ \\
u24g & 6 hours & $2^{3} \cdot 3^{1}=24$ \\
u32g & 8 hours & $2^{5} \cdot 3^{0}=32$ \\
u48g & 12 hours & $2^{4} \cdot 3^{1}=48$ \\
u96g & 24 hours & $2^{5} \cdot 3^{1}=96$ \\
\hline
\end{tabular}

The variation of electron density in ionosphere is strongly related to solar sunspots cycle (around 11 years), as well as geomagnetic activity. In order to carry out a comprehensive analysis, one and a half solar cycle (from 2002 to 2019) uqrg GIMs, computed by UPC-IonSAT, were downloaded from ftp: //newg1.upc.es/upc_ionex. Solar and geomagnetic index (F10.7 and Ap, respectively) were downloaded from ftp://ftp.swpc.noaa.gov/pub/indices/ and ftp://ftp.gfz-potsdam.de/pub/home/obs/kp-ap/wdc/ to analyse the behaviour of assessment results under different solar radiation and geomagnetic conditions. 


\section{VTEC reference-Jason}

Jason was the follow-on to the well-known TOPEX/Poseidon mission, and was composed of a series of satellites: Jason-1, Jason-2, Jason-3. These satellites were joint missions of NASA-CNES and started to afford official service from 2002 to now $[21,15]$. Altimeters of Jason continuously send and receive bounced dual-frequency signals for measuring sea surface height from a orbital height of around $1336 \mathrm{~km}$. VTEC along the ray path from satellite to the sea surface, as a by-product of these measurements, can be derived from $\mathrm{Ku}$-band $(13.575 \mathrm{GHz})$ and $\mathrm{C}$-band $(5.3 \mathrm{GHz})$ observations. It is provided as a ionospheric correction of $\mathrm{Ku}$-band in Jason altimeter data file [22]. The extraction of VTEC, in TECUs, from altimeter data can be realized by Equation 1 (as described in [14]).

$$
V T E C=-\frac{f_{K u}^{2} \cdot \Delta r_{K u-i o n}}{40.3 \cdot 10^{16}}
$$

where $\Delta r_{K u-i o n}$ is the $\mathrm{Ku}$-band ionospheric correction, in meters, obtained from file; $f_{K u}$ is the frequency, in $\mathrm{Hz}$, of $\mathrm{Ku}$-band.

Subsequently, the direct VTEC is smoothed by a sliding window of 16 seconds to mitigate the impact of noise from altimeter measurements [13]. Since the altitude of altimeter is approximately $1336 \mathrm{~km}$, TEC of plasmasphere above this altitude is ignored (up to few TECU). In addition, a systematic bias of VTEC-Jason measurement, ranging from 2 to 5 TECU (as described in [1]). In this context, standard deviation (STD) of the difference between VTEC-Jason and VTEC-GIM is discussed below, since standard deviation can significantly mitigate the effects of these sources of error. Despite these limited errors, VTEC Jason is an accurate, reliable and independent reference measurement source which is relatively more suitable for GIM assessment on the ocean [13, 20].

Hence, we took VTEC-Jason as the main reference for assessment of GIMs. The original TEC data from Jason were derived every second along the satellite orbit, while the temporal and spatial resolution of uqrg is 15 minutes and $5^{\circ} \times 2.5^{\circ}$ in longitude and latitude, respectively. Therefore, the most commonly used grid interpolation method in GIM was adopted [24]. As a result, a direct comparison between VTEC-Jason and VTEC-GIM can be obtained.

The observation number of VTEC-Jason per day during the period of 20022019 is shown in Figure 1. Despite some fluctuation, the average number of observations per day is higher than 30000, which is sufficient for GIMs validation.

The daily statistics for VTEC-Jason assessment was based on the difference between VTEC-Jason and VTEC-GIM. Two main statistics were involved in the section 5: bias and standard deviation. The computation of these two statistics was as Equation 2 and 3:

$$
\text { Bias }=\frac{\sum_{i=1}^{N}\left(V T E C_{J a s o n, i}-V T E C_{G I M, i}\right)}{N}
$$




$$
S T D=\sqrt{\frac{\sum_{i=1}^{N}\left(V T E C_{J a s o n, i}-V T E C_{G I M, i}-B i a s\right)^{2}}{N-1}}
$$

where $V T E C_{J a s o n, i}$ and $V T E C_{G I M, i}$ are VTEC extracted from Jason and GIM, respectively; $N$ is the number of involved observations; $i$ identifies the observation.

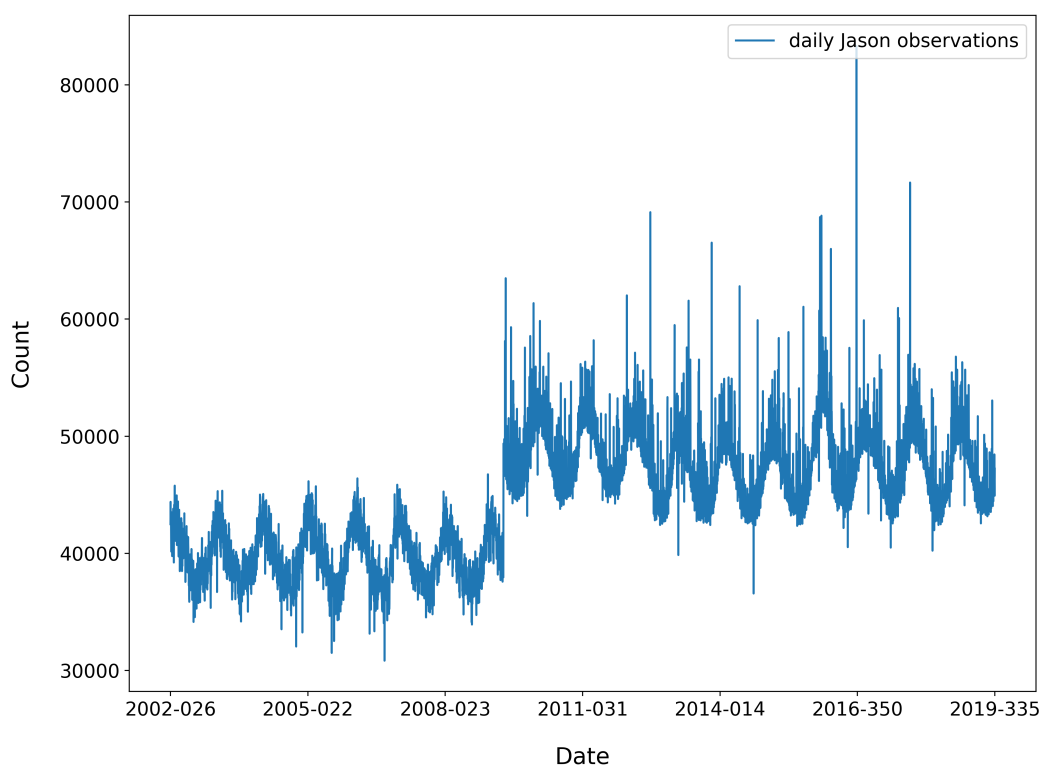

Fig. 1 The number of observations of Jason altimeters taken as reference in this work, from day 26 of 2002 to day 335 of 2019 .

In addition, the influence of the solar geoeffective (mostly EUV) radiation on the formation of ionosphere in a specific region can be generally represented by the introduction of daily average VTEC over this area. The daily mean VTEC is calculated by averaging the VTEC-Jason of specific region per day as in Equation 4:

$$
\text { daily mean } V T E C=\frac{\sum_{i=1}^{N} V T E C_{J a s o n, i}}{N}
$$

where the symbols are as same as Equation 2.

\section{4 dSTEC reference}

The difference of ionospheric linear combination $L_{I}=L_{1}-L_{2}$ obtained from GPS carrier phase observation from the same transmitter-receiver at a given 
time regarding the time with maximum elevation above the horizon (within a phase-continuous arc) is another reference for GIM. This reference is more accurate than the altimeter VTEC for the assessment of GIM, and has been widely applied in the assessment of ionospheric models $([22,13])$. In this paper, the dSTEC assessment was performed based on the dSTEC error, by following Equation 5 [13]:

$$
\begin{aligned}
\delta & =\Delta S_{G P S}-\Delta S_{G I M} \\
& =\left(S(t)_{G P S}-S\left(t_{E_{\max }}\right)_{G P S}\right)-\left(S(t)_{G I M}-S\left(t_{E_{\max }}\right)_{G I M}\right) \\
& =\frac{1}{\alpha}\left(L_{I}(t)_{G P S}-L_{I}\left(t_{E_{\max }}\right)_{G P S}\right)-M \cdot\left(V(t)_{G I M}-V\left(t_{E_{\max }}\right)_{G I M}\right)
\end{aligned}
$$

where $S$ and $V$ are STEC and VTEC, respectively; $E_{\text {max }}$ is the maximum value of elevation within a continuous phase-arc; $\alpha \simeq 40.3$ in S.I. units; $L_{I}=L_{1}-L_{2}$ is the difference of carrier phase observation (the small carrier phase windup can be corrected in advance).

According to previous research, the accuracy of dSTEC measurement from GPS observation is much higher than 0.1 TECU [13]. Such dSTEC can be considered as an excellent reference for GIM assessment. In this article, 59 homogeneously distributed GNSS receivers that were not involved in the generation of uqrg GIM, were selected for the assessment during four days in 2015: 082, 146, 280, 330 (similarly like in [22], see Figure 2).

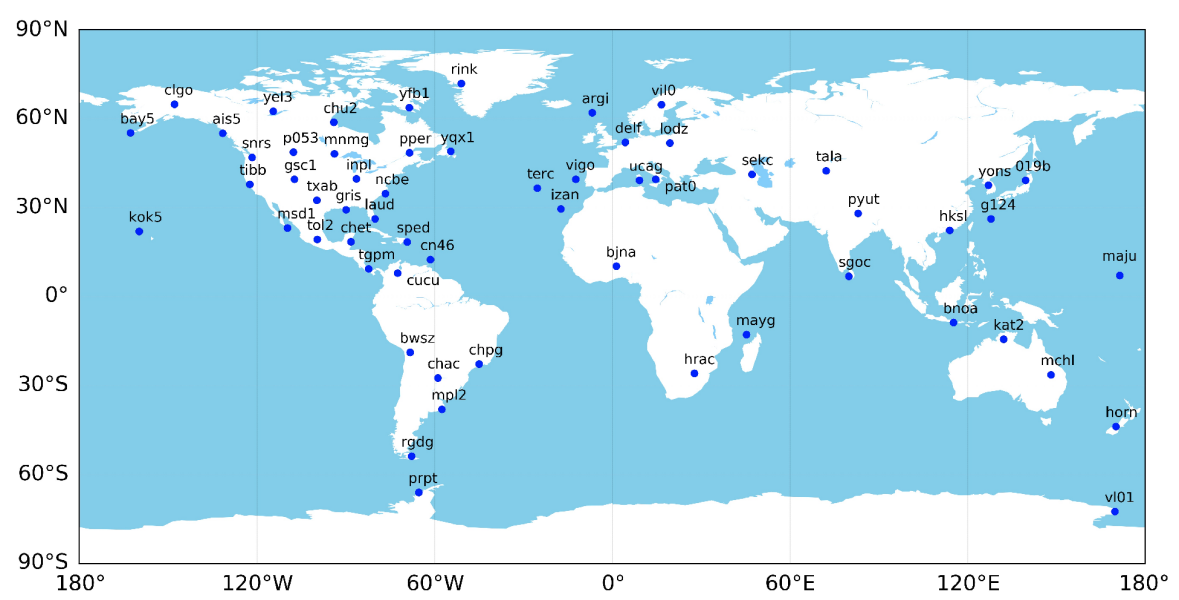

Fig. 2 Distribution of selected 59 GNSS receivers for dSTEC-GPS assessment during the analyzed days $082,146,280$ and 330 of year 2015 .

The statistics for assessment were based on dSTEC error $\delta$. Three statistics were involved in section 5: RMS, standard deviation and relative error. The computation of these three statistics followed Equation 6, 7 and 8 :

$$
R M S_{\delta}=\sqrt{\frac{\sum_{i=1}^{N}\left(\delta_{i}\right)^{2}}{N}}
$$




$$
\begin{gathered}
S T D=\sqrt{\frac{\sum_{i=1}^{N}\left(\delta_{i}-\mu\right)^{2}}{N-1}} \\
\text { Relative error }=\frac{R M S_{\delta}}{R M S_{\Delta S_{G P S}}}
\end{gathered}
$$

where $\delta_{i}$ is the dSTEC error at epoch i; $\mu$ is the mean value of $\delta_{i} ; R M S_{\triangle S_{G P S}}$ is the RMS of observed dSTEC-GPS.

\section{Results and analysis}

In this section the VTEC from GIMs of different temporal resolutions are compared with VTEC from Jason-altimeter data during 2002 to 2019. The assessment results were based on the difference VTEC-GIM and VTEC-Jason and were analysed in detail considering the impact of solar flux, geomagnetic activity, and geographical location.

\subsection{Overall results of VTEC-Jason assessment}

As it can be seen from Figure 3, the standard deviation of the difference between VTEC-GIM and VTEC-Jason shared a similar tendency with solar flux index (F10.7) and daily mean VTEC during one and a half solar cycle. Such error is approximately sorted from higher to lower time resolution, being such difference more evident around solar cycle peaks (2002 and 2014), and when the geomagnetic activity (Ap) is higher, as it might be expected. Figure 4 clearly shows a high linear correlation between the daily mean VTEC and F10.7 index, which is consistent with the corresponding results shown in [11]. The standard deviation of the difference between VTEC-GIM and VTECJason was linearly related except for some fluctuations (mostly share with Ap variation) and the error of the twelve GIMs tends to be larger at higher mean VTEC. However, the difference was not noticeable for mean VTEC equals to 37 TECU (with very high Ap, i.e. geomagnetic activity) and higher (aroung the solar cycle peak), which represent a huge challenge to all GIMs. In addition, it should be noted that when the value of daily mean VTEC was not high (less than 20), the standard deviation was mainly related with solar activity index F10.7 but it was dependent on both F10.7 and Ap index at high daily mean VTEC.

With the increase of Ap index in Figure 5, the standard deviation of difference between VTEC-GIM and VTEC-Jason tended to present a high variability and the difference between the 12 GIMs was less stable than in Figure 4. At the beginning of $\mathrm{x}$-axis (low daily Ap index), the evolution of standard deviation of VTEC-GIM versus VTEC-Jason was quite smooth. After the daily Ap index increased to 42 , the response of this evolution to the variation of solar flux (F10.7) and daily mean VTEC became obvious. And most fluctuations 


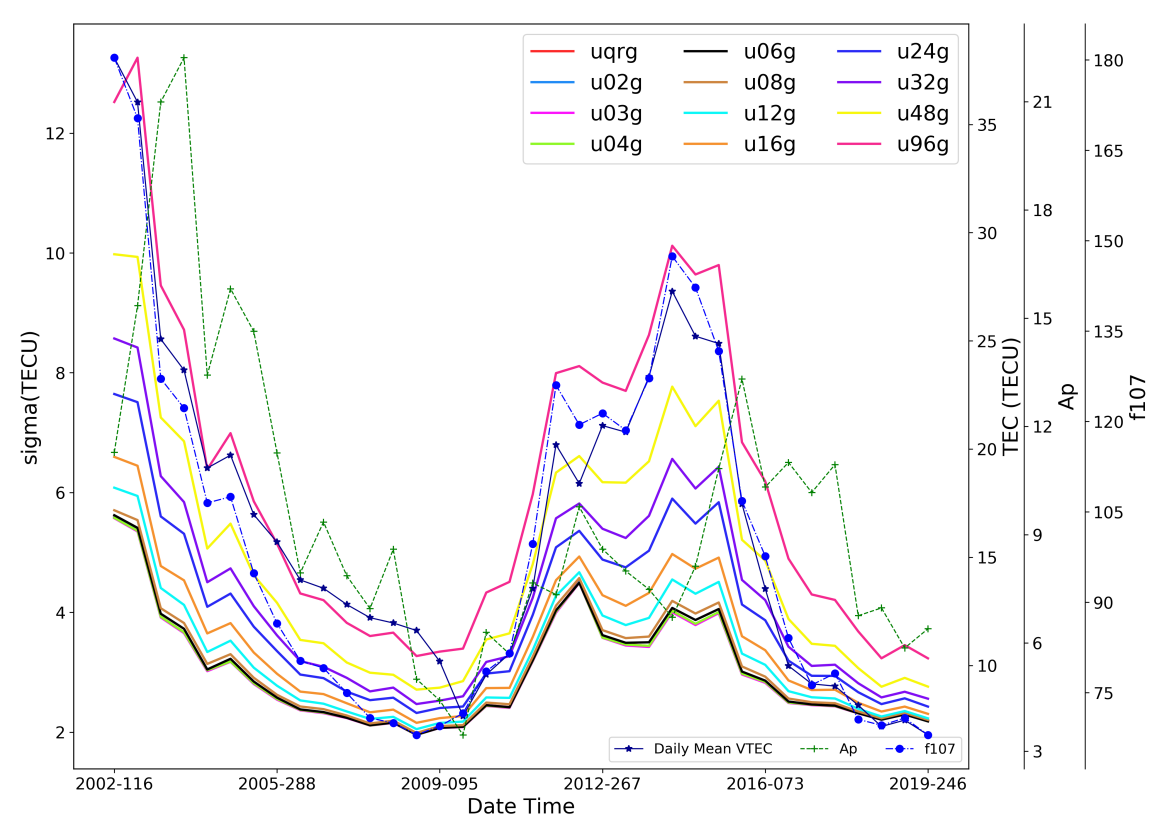

Fig. 3 The evolution of standard deviation of the discrepancy of GIM VTEC vs. measured altimeter VTEC, in TECUs, from day 26 of 2002 to day 335 of 2019, represented simultaneously to Ap geomagnetic activity and F10.7 solar flux indices (green crosses and blue bullets, respectively).

of standard deviation were coincident with solar flux and daily mean VTEC fluctuations. In addition, the peak value of standard deviation kept increasing under intense geomagnetic activity.

The histograms in Figure 6 clearly indicates the discrepancy of these GIMs. The $\mathrm{x}$-axis of histograms represents the value of standard deviation of difference between VTEC-GIM and VTEC-Jason, and y-axis is the corresponding counts of values. The distribution and statistics of first four GIMs (from uqro to $\mathrm{u} 04 \mathrm{~g}$ ) were pretty similar. However, a noticeable difference can firstly be seen in $\mathrm{u} 06 \mathrm{~g}$ ( 1.5 hours) and u08g (2 hours) where the maximum value of $y$-axis which corresponds to the count of standard deviation declined to less than 100. The magnitude of median value increased from 5.2 (uqrg-u04g) to $5.3(\mathrm{u} 06 \mathrm{~g})$ and $5.4(\mathrm{u} 08 \mathrm{~g})$. In addition, the shape of distribution for remaining GIMs started to shift with lower time resolution (from $\mathrm{u} 12 \mathrm{~g}$ to $\mathrm{u} 96 \mathrm{~g}$ ) and the peak value of y-axis also decreased.

To make a comparison, the validation results of different latitudes were binned as shown in Table 2. Hereafter we will refer to latitudinal band id. For example, the validation results between 50 to 70 degree (actually 66 degree, coinciding with the altimeter orbital inclination) will be labelled as those of 60 degree latitudinal band.

A summary of statistics is shown in Table 3 and Table 4, taking into account 


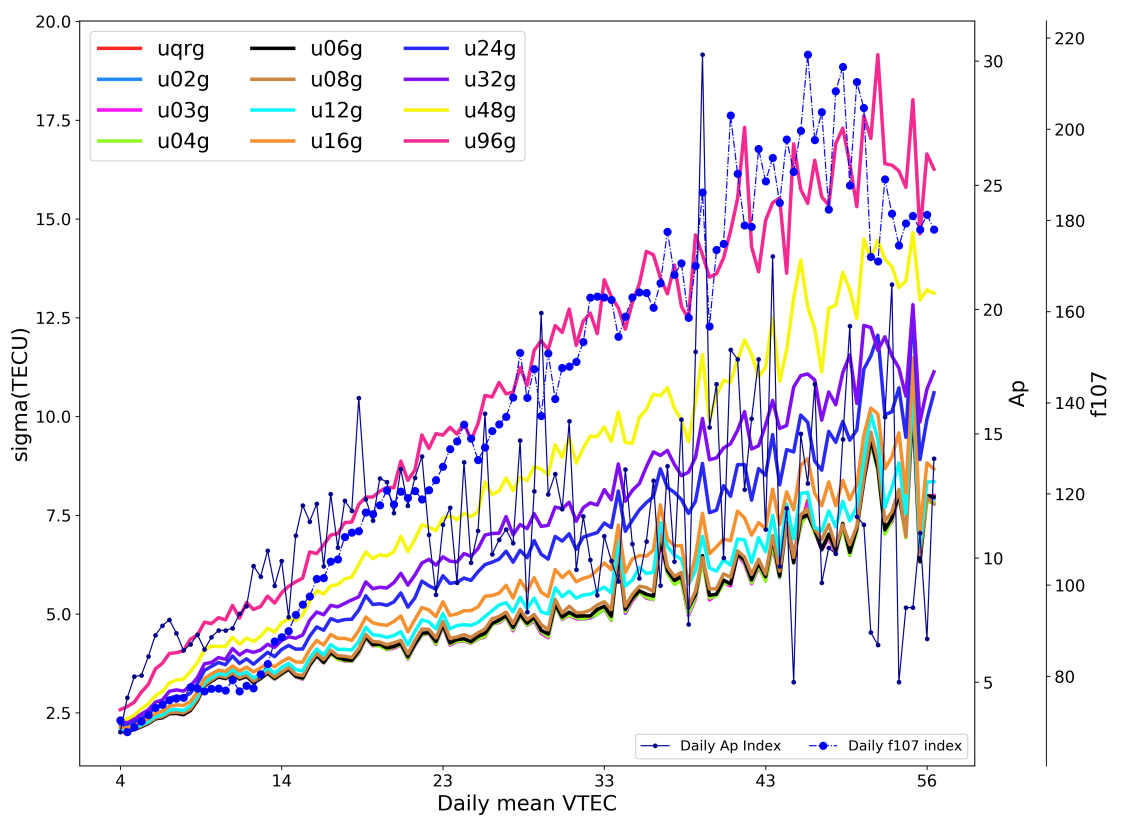

Fig. 4 Daily standard deviation of the discrepancy of GIM VTEC vs. measured altimeter VTEC, in TECUs, represented versus daily mean VTEC from day 26 of 2002 to day 335 of 2019 .

Table 2 Range of latitudinal bands studied in detail in this work.

\begin{tabular}{cc}
\hline Latitudinal band / degree & Latitudinal range / degree \\
\hline 60 & {$[50,66)$} \\
40 & {$[30,50)$} \\
20 & {$[10,30)$} \\
0 & {$[-10,10)$} \\
-20 & {$[-30,-10)$} \\
-40 & {$[-50,-30)$} \\
-60 & {$[-66,-50)$} \\
\hline
\end{tabular}

that the impact of the altimeter VTEC measurements error is at the level of 0.1 TECU (see for instance the Figure 12 right-hand plot in [12]). Firstly, the Table 3 records the performance of 12 GIMs at seven latitudinal intervals (hereinafter latitudinal bands) and corresponding average value. The bias of VTEC-Jason minus VTEC-GIM get a minimum at equator and subsequently increases towards polar region with a maximum, close to zero, at 60 degree latitudinal band. This is consistent, regardless of any potential VTEC-Jason instrumental calibration bias ([1]), with the typical latitudinal distribution of the small electron content located above Jason- and below GPS- satellites. In addition, the bias of southern hemisphere was larger than that of northern hemisphere except for mid latitude (-40 and 40 latitudinal band). The wellknown equatorial anomaly peaks and sparse distribution of GNSS receivers at 


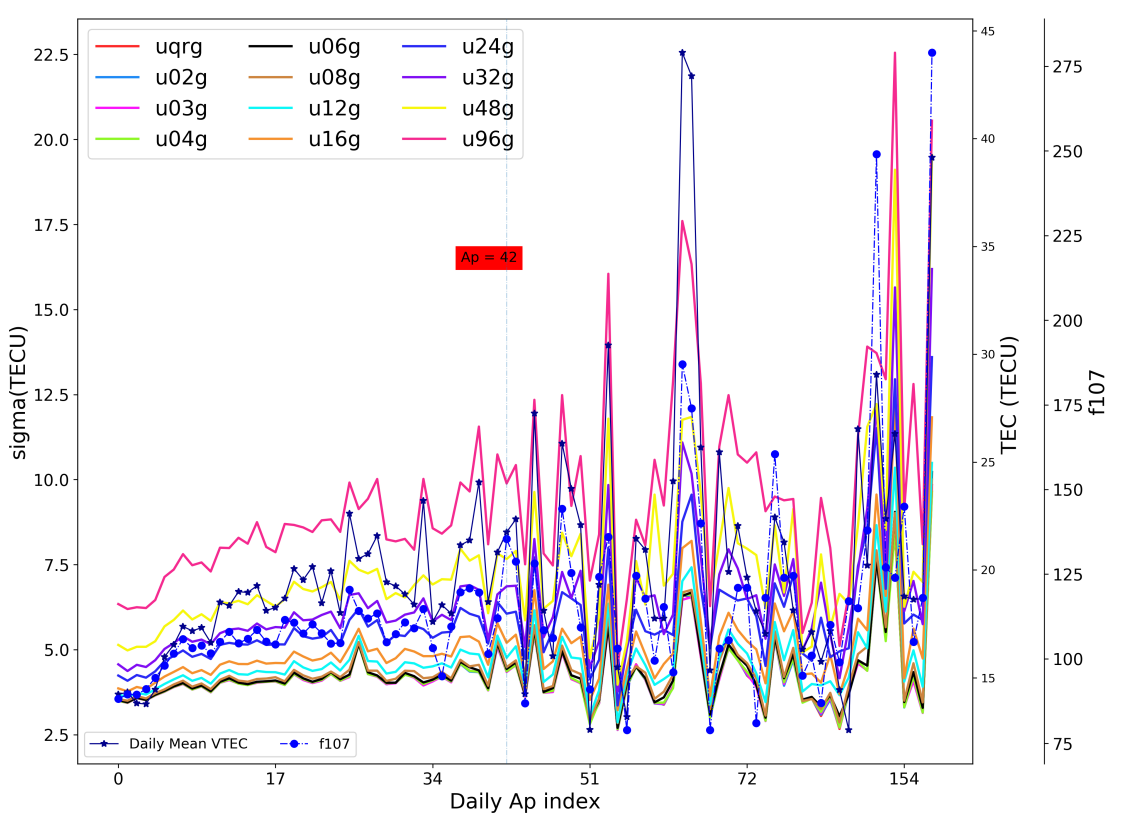

Fig. 5 Daily standard deviation of the discrepancy of GIM VTEC vs. measured altimeter VTEC, in TECUs, represented versus daily Ap index from day 26 of 2002 to day 335 of 2019 .

southern hemisphere may account for this result.

Table 3 Bias of GIM versus Jason VTEC in TECUs, from day 26 of 2002 to day 335 of 2019, computed as corresponding average of Jason VTEC minus GIM VTEC, at different latitudinal bands in TECUs.

\begin{tabular}{ccccccccc}
\hline GIM & $-60^{\circ}$ & $-40^{\circ}$ & $-20^{\circ}$ & $0^{\circ}$ & $20^{\circ}$ & $40^{\circ}$ & $60^{\circ}$ & Overall \\
\hline $\mathrm{uqrg}$ & -0.1 & -0.6 & -1.4 & -1.7 & -1.1 & -1.0 & -0.1 & -0.9 \\
$\mathrm{u} 02 \mathrm{~g}$ & -0.1 & -0.6 & -1.4 & -1.7 & -1.1 & -1.0 & -0.1 & -0.9 \\
$\mathrm{u} 03 \mathrm{~g}$ & -0.1 & -0.6 & -1.4 & -1.7 & -1.1 & -1.0 & -0.1 & -0.9 \\
$\mathrm{u} 04 \mathrm{~g}$ & -0.1 & -0.6 & -1.4 & -1.7 & -1.1 & -1.0 & -0.1 & -0.9 \\
$\mathrm{u} 06 \mathrm{~g}$ & -0.1 & -0.6 & -1.4 & -1.7 & -1.1 & -1.0 & -0.1 & -0.9 \\
$\mathrm{u} 08 \mathrm{~g}$ & -0.1 & -0.6 & -1.4 & -1.7 & -1.1 & -0.9 & -0.1 & -0.9 \\
$\mathrm{u} 12 \mathrm{~g}$ & -0.1 & -0.6 & -1.4 & -1.7 & -1.1 & -0.9 & -0.1 & -0.9 \\
$\mathrm{u} 16 \mathrm{~g}$ & -0.1 & -0.6 & -1.4 & -1.7 & -1.1 & -0.9 & -0.1 & -0.9 \\
$\mathrm{u} 24 \mathrm{~g}$ & -0.0 & -0.6 & -1.5 & -1.5 & -1.2 & -0.9 & -0.1 & -0.9 \\
$\mathrm{u} 32 \mathrm{~g}$ & -0.1 & -0.7 & -1.4 & -1.4 & -1.2 & -1.0 & -0.2 & -0.9 \\
$\mathrm{u} 48 \mathrm{~g}$ & -0.1 & -0.5 & -1.0 & -1.2 & -1.6 & -1.1 & -0.2 & -0.8 \\
$\mathrm{u} 96 \mathrm{~g}$ & 0.3 & 0.8 & -2.0 & -1.5 & -1.2 & -1.6 & -0.3 & -0.7 \\
\hline
\end{tabular}

Table 4 shows the records of standard deviation which also reached maximum and minimum at equator and high latitude bands, respectively. As it can be seen in Table 4, there are some values marked with bold font for each column. 

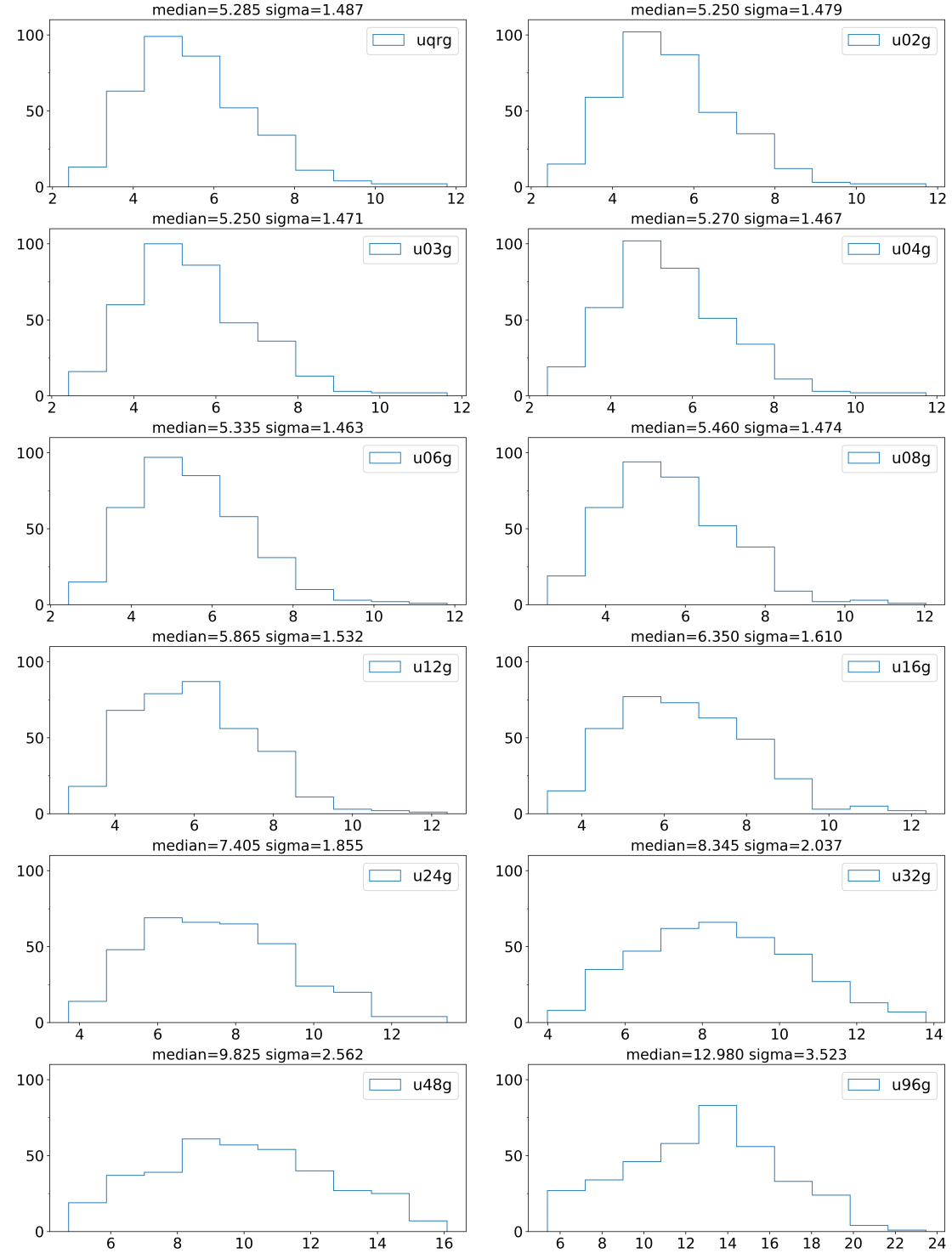

Fig. 6 The histograms of standard deviation of the discrepancy of GIM VTEC vs. measured altimeter VTEC, in TECUs, from day 26 of 2002 to day 335 of 2019. 
Table 4 Standard deviation of GIM versus Jason VTEC in TECUs, from day 26 of 2002 to day 335 of 2019, at different latitudinal bands in TECUs. The values showing a first increase of 0.1 TECU in the standard deviation in each given latitudinal band are represented in bold.

\begin{tabular}{ccccccccc}
\hline GIM & $-60^{\circ}$ & $-40^{\circ}$ & $-20^{\circ}$ & $0^{\circ}$ & $20^{\circ}$ & $40^{\circ}$ & $60^{\circ}$ & Overall \\
\hline $\mathrm{uqrg}$ & 3.7 & 3.3 & 4.2 & 4.7 & 4.4 & 2.9 & 2.7 & 3.9 \\
$\mathrm{u} 02 \mathrm{~g}$ & 3.7 & 3.3 & 4.2 & 4.7 & 4.4 & 2.9 & 2.7 & 3.9 \\
$\mathrm{u} 03 \mathrm{~g}$ & 3.7 & 3.3 & 4.2 & 4.7 & 4.4 & 2.9 & 2.7 & 3.9 \\
$\mathrm{u} 04 \mathrm{~g}$ & 3.7 & 3.3 & 4.2 & 4.7 & 4.4 & 2.9 & 2.7 & 3.9 \\
$\mathrm{u} 06 \mathrm{~g}$ & 3.7 & 3.3 & 4.2 & 4.7 & $\mathbf{4 . 5}$ & 2.9 & 2.7 & 3.9 \\
$\mathrm{u} 08 \mathrm{~g}$ & 3.7 & 3.3 & $\mathbf{4 . 3}$ & $\mathbf{4 . 8}$ & 4.6 & 2.9 & 2.7 & 3.9 \\
$\mathrm{u} 12 \mathrm{~g}$ & 3.7 & $\mathbf{3 . 4}$ & 4.6 & 5.0 & 4.9 & $\mathbf{3 . 0}$ & $\mathbf{2 . 8}$ & $\mathbf{4 . 1}$ \\
$\mathrm{u} 16 \mathrm{~g}$ & $\mathbf{3 . 8}$ & 3.5 & 4.9 & 5.3 & 5.3 & 3.1 & 2.8 & 4.3 \\
$\mathrm{u} 24 \mathrm{~g}$ & 4.0 & 3.7 & 5.7 & 5.9 & 6.1 & 3.4 & 3.0 & 4.8 \\
$\mathrm{u} 32 \mathrm{~g}$ & 4.2 & 4.0 & 6.3 & 6.3 & 6.8 & 3.7 & 3.1 & 5.2 \\
$\mathrm{u} 48 \mathrm{~g}$ & 4.9 & 4.6 & 7.3 & 6.8 & 7.6 & 4.0 & 3.3 & 5.9 \\
$\mathrm{u} 96 \mathrm{~g}$ & 5.5 & 6.1 & 10.3 & 8.1 & 9.2 & 4.3 & 3.5 & 7.4 \\
\hline
\end{tabular}

The standard deviation of GIM TEC vs. altimeter VTEC start to increase with lower temporal resolution after these values in bold font. In this context, the standard deviation of each latitudinal band can be divided into two groups: above the value in bold font, below the value in bold font. In general, the variation of ionosphere is typically assumed as linear between two consecutive TEC maps in sun-fixed reference frame for few hours ([24]). This assumption is assessed and quantified in Table 4, where the values up to the bold value in each band remained the same at 0.1 TECU level. However, the ionosphere dynamics is more complex and may cause different variations of electron density. This is reflected in the values from the bold value up, in each latitudinal band, which started to significantly increase. Since the GIM is a global description of ionosphere, we should take into account all the latitudinal bands. Therefore, we can say that the performance against the VTEC altimeter does not degrade significantly up to 1 hour time resolution or less (GIM with id. uqrg- $\mathrm{u} 04 \mathrm{~g}$ ). And this is not the case for time resolutions of 1.5 hours and up (GIM id. u06g-u96g), with evident degradation in the GIM performance.

\subsection{Results of VTEC-Jason assessment at different latitudes}

To compare the performance of the different time-resolution GIMs at different latitudes, the time evolution of standard deviation of VTEC GIM vs. VTEC altimeter previously shown in Figure 3 was splited into seven plots in Figure 7, corresponding to the seven latitudinal intervals of 20 degrees length each. The upper left figure presents the time series of standard deviation of VTEC-GIM versus VTEC-Jason at 60 degree latitudinal band (ranging from 50 degrees to 66 degrees, the Jason orbital angle), and the upper right picture shows the time series at corresponding -60 degree latitudinal band. Subsequently the remaining figures shows the time series corresponding to latitudinal intervals centered at 40, -40,20,-20 and 0 degrees. The evolution of standard deviation 
of VTEC-GIM versus VTEC-Jason seems to be mainly affected by solar and geomagnetic activity (characterized by F10.7 and Ap index respectively). Although the evolution of standard deviation in these seven pictures resembled the evolution of Figure 3, some differences can be seen between different latitudinal bands.

In Figure 7, the standard deviation of the GIM discrepancy vs. altimeter VTEC measurements declined at different latitudes during the period from 2002 to 2009 and started to rise after 2009, accordingly with mean VTEC and F10.7 evolution during the solar cycle. This happens in particular at the next time span of 7 years (from 2009 to 2016) when all the time series of standard deviation achieved two peak values. Three latitudinal bands of southern hemisphere encountered a sharp increase around 2012 (the first peak) and a slight growth around 2014 (the second peak). However, the remaining bands of northern hemisphere suffered a contrary tendency compared with southern hemisphere. The time series of southern hemisphere was in accordance with the variation of VTEC value and F10.7 index, which was reasonable under normal condition. This phenomenon may be caused by the geomagnetic activity. As we can see from figures, the time series of daily Ap index (the green line) indicates that the geomagnetic field suffered significant disturbances at 2012, while the geomagnetic field was quiet around 2014. This coincidence suggests that the disturbed geomagnetic field complicates the modelling of ionosphere and it induces a severe impact on southern hemisphere, where the distribution of receivers is sparse, with a higher geomagnetic field asymmetry of the south pole vs. the north pole [19]. In addition, the discrepancies between highresolution GIMs (uqrg-u08g) of different latitudinal bands were not identical. The discrepancies at northern hemisphere are considerably larger than those at southern hemisphere. An apparent difference among u06g, u08g and highresolution GIMs (uqrg, u02g, u03g, u04g) can be seen in 20 degree latitudinal band.

Similarly, Figure 8 and Figure 9 presented the performance vs mean observed VTEC and vs. Ap daily index as different sort criteria for the seven latitudinal bands. In Figure 8, while the range of y-axis (i.e. the standard deviation of the GIM VTEC discrepancy vs the altimeter VTEC) is larger in southern hemisphere than in the northern hemisphere, the range of daily mean VTEC at six latitudinal bands is approximately symmetrical and reached a maximum at 0 degrees latitudinal band. The discrepancy among high-resolution GIMs (uqrg to $\mathrm{u} 08 \mathrm{~g}$ ) was more apparent at northern hemisphere than at southern hemisphere and was larger at lower latitudinal band. As shown in the image of 20 degree latitudinal band, the difference among $\mathrm{u} 06 \mathrm{~g}$, u08g and high-resolution GIMs (uqrg, u02g, u03g, u04g) was obvious. However, that relative discrepancy was smaller at equator, coinciding with its higher standard deviation values of the GIM VTEC discrepancy vs. the altimeter, compared with other latitudinal bands.

As can be seen in Figure 9, the magnitude of standard deviation of VTECGIM versus VTEC-Jason at southern hemisphere appeared to be slightly 

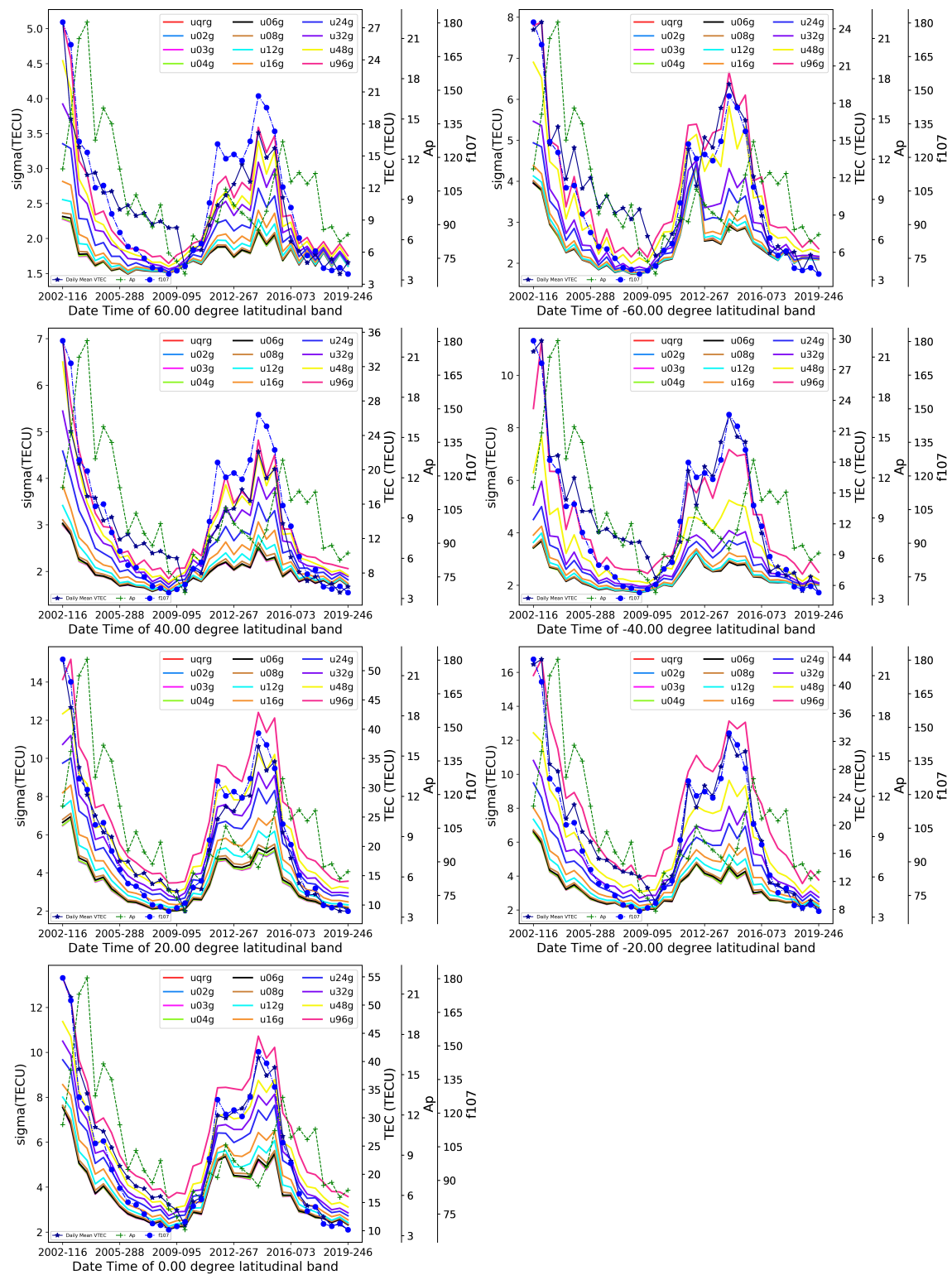

Fig. 7 The evolution of standard deviation of the discrepancy between GIM VTEC and measured altimeter VTEC, in TECUs, from day 26 of 2002 to day 335 of 2019, at different latitudinal bands. 

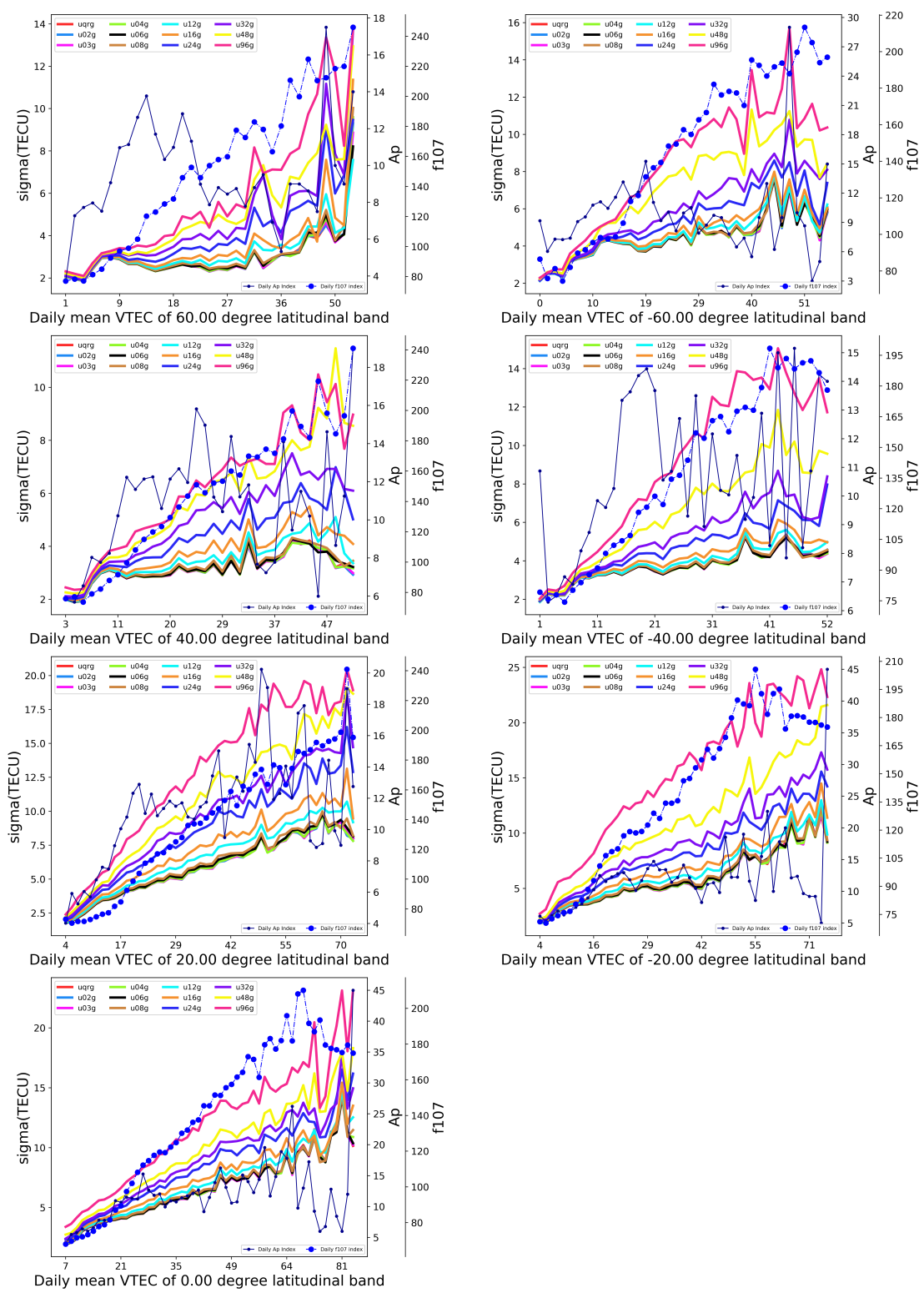

Daily mean VTEC of -20.00 degree latitudinal band

Fig. 8 The daily standard deviation of the discrepancy of GIM VTEC vs. measured altimeter VTEC, in TECUs, is represented versus daily mean VTEC from day 26 of 2002 to day 335 of 2019, at different latitudinal bands. 

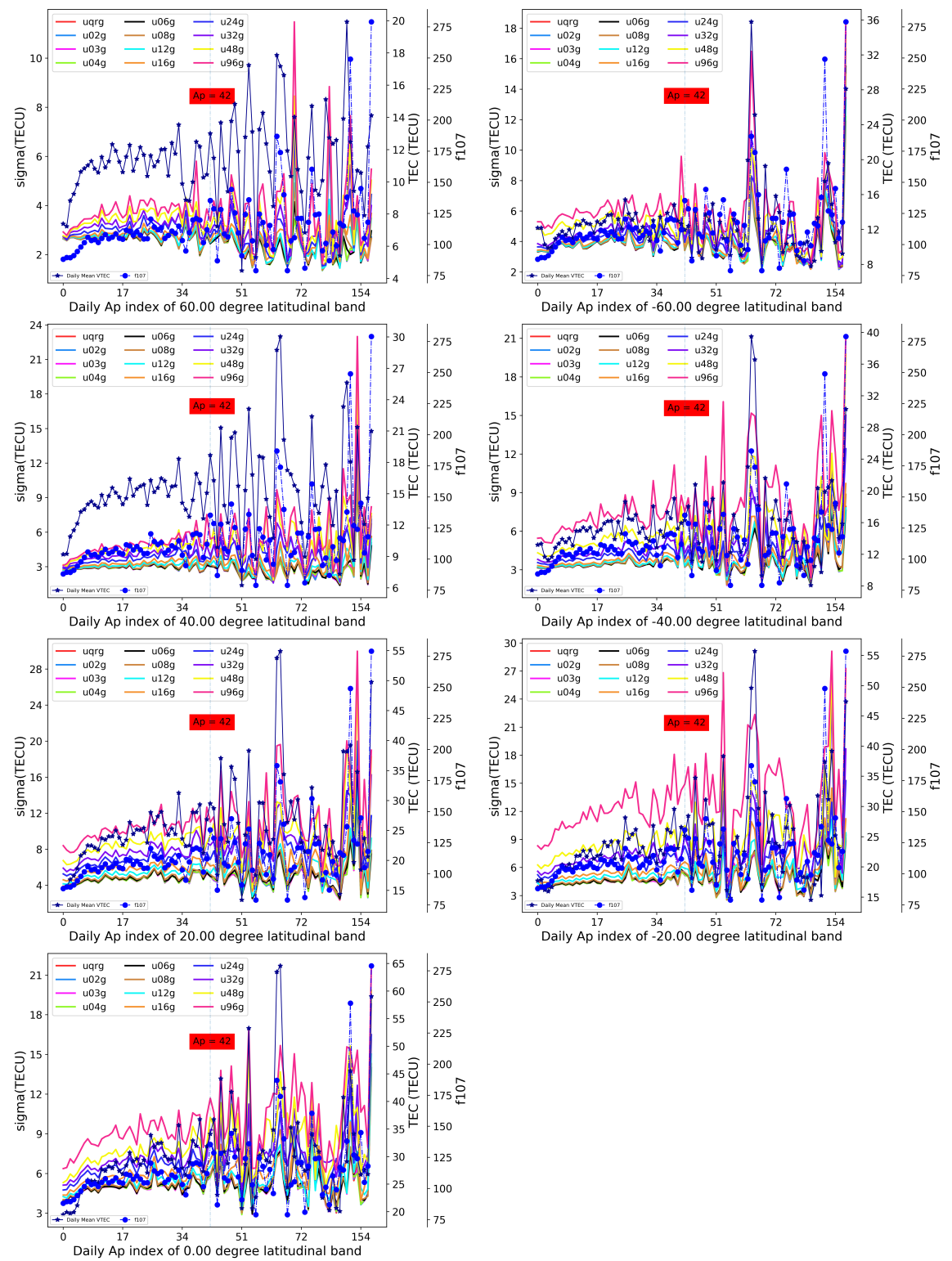

Fig. 9 The daily standard deviation of the discrepancy of GIM VTEC vs. measured altimeter VTEC, in TECUs, is represented versus daily Ap index from day 26 of 2002 to day 335 of 2019 , at different latitudinal bands 
higher than at northern hemisphere, and this may be related with the poor accuracy of GIM at southern hemisphere [11]. And the evolution of standard deviation at different latitudinal bands was more relevant with daily mean VTEC at corresponding bands rather than solar activity index F10.7, since the solar index F10.7 represented the entire influence of solar activity.

In Figure 4, Figure 5, Figure 8, and Figure 9, the difference among GIMs with various temporal resolution was more noticeable at high Ap index and high mean VTEC. In order to obtain a clear comparison of standard deviation of VTEC-GIM versus VTEC-Jason at solar cycle maximum conditions and geomagnetic activity, 90\% was selected as reference threshold, which means $10 \%$ of remaining results, above such threshold, were selected for comparison. Thresholds of Ap index and daily mean VTEC were $20(2 \mathrm{nT})$ and 28.95 (TECU) respectively and the results of standard deviation of VTEC-GIM versus VTEC-Jason after these thresholds has been selected. The summary of these results are shown in Table 5 and Table 6 . It should be noted that the magnitude of difference among GIMs was more conspicuous near the equator and that difference in northern hemisphere was larger than in the southern hemisphere. For example, the differences between uqrg and u08g in Table 6 at seven latitudinal bands were $0.1,0.1,0.3,0.3,0.4,0.2,0.1$ TECUs respectively.

Table 5 Standard deviation of VTEC-GIM versus VTEC-Jason, in TECUs, at different latitudinal bands for days at the top $10 \%$ of daily mean VTEC, from day 26 of 2002 to day 335 of 2019. The values showing a first increase of $0.1 \mathrm{TECU}$ in the standard deviation in each given latitudinal band are represented in bold.

\begin{tabular}{ccccccccc}
\hline GIM & $-60^{\circ}$ & $-40^{\circ}$ & $-20^{\circ}$ & $0^{\circ}$ & $20^{\circ}$ & $40^{\circ}$ & $60^{\circ}$ & Overall \\
\hline uqrg & 5.0 & 4.1 & 5.8 & 6.3 & 6.2 & 3.6 & 3.4 & 5.6 \\
u02g & 5.0 & 4.1 & 5.8 & 6.3 & 6.2 & 3.6 & 3.4 & 5.6 \\
u03g & 5.0 & 4.1 & 5.8 & 6.3 & 6.2 & 3.6 & 3.4 & 5.6 \\
u04g & 5.0 & 4.1 & 5.8 & 6.3 & 6.2 & 3.6 & 3.4 & 5.6 \\
u06g & 5.0 & 4.1 & $\mathbf{5 . 9}$ & 6.3 & $\mathbf{6 . 3}$ & 3.6 & $\mathbf{3 . 5}$ & $\mathbf{5 . 7}$ \\
u08g & $\mathbf{5 . 1}$ & $\mathbf{4 . 2}$ & 6.0 & $\mathbf{6 . 5}$ & 6.5 & $\mathbf{3 . 7}$ & 3.6 & 5.8 \\
u12g & 5.3 & 4.4 & 6.6 & 6.8 & 7.1 & 4.0 & 3.8 & 6.2 \\
u16g & 5.6 & 4.8 & 7.2 & 7.3 & 7.8 & 4.4 & 4.2 & 6.7 \\
u24g & 6.4 & 5.6 & 8.5 & 8.2 & 9.2 & 5.2 & 5.1 & 7.7 \\
u32g & 7.2 & 6.4 & 9.6 & 8.9 & 10.3 & 6.1 & 5.9 & 8.6 \\
u48g & 9.1 & 8.2 & 11.6 & 9.6 & 11.9 & 7.2 & 6.8 & 10.2 \\
u96g & 10.9 & 12.5 & 16.7 & 11.7 & 14.4 & 7.9 & 8.2 & 13.5 \\
\hline
\end{tabular}

According to the criteria used in Table 4, the results of Table 5 and Table 6 can be divided into two groups: uqro-u04g and u06g-u96g. The accuracy within the first group, the GIMs with the highest time resolution up to 1 hour, is comparable, while the accuracy within the second group of GIMs (with time resolutions from 1.5 to 24 hours) decreases following the decrease of temporal resolution. 
Table 6 Standard deviation of VTEC-GIM versus VTEC-Jason, in TECUs, at different latitudinal bands for days at the top $10 \%$ of daily Ap index, from day 26 of 2002 to day 335 of 2019. The values showing a first increase of 0.1 TECU in the standard deviation in each given latitudinal band are represented in bold.

\begin{tabular}{ccccccccc}
\hline GIM & $-60^{\circ}$ & $-40^{\circ}$ & $-20^{\circ}$ & $0^{\circ}$ & $20^{\circ}$ & $40^{\circ}$ & $60^{\circ}$ & Overall \\
\hline uqrg & 4.4 & 4.2 & 5.6 & 6.0 & 5.5 & 3.2 & 2.6 & 4.9 \\
u02g & 4.4 & 4.2 & 5.6 & 6.0 & 5.5 & 3.2 & 2.6 & 4.9 \\
u03g & 4.4 & 4.2 & 5.6 & 6.0 & 5.5 & 3.2 & 2.6 & 4.9 \\
u04g & 4.4 & 4.2 & 5.6 & 6.0 & 5.5 & 3.2 & 2.6 & 4.9 \\
u06g & $\mathbf{4 . 5}$ & 4.2 & $\mathbf{5 . 7}$ & $\mathbf{6 . 1}$ & $\mathbf{5 . 7}$ & $\mathbf{3 . 3}$ & $\mathbf{2 . 7}$ & $\mathbf{5 . 0}$ \\
u08g & 4.5 & $\mathbf{4 . 3}$ & 5.9 & 6.3 & 5.9 & 3.4 & 2.7 & 5.1 \\
u12g & 4.6 & 4.5 & 6.4 & 6.6 & 6.6 & 3.8 & 2.9 & 5.4 \\
u16g & 4.7 & 4.8 & 7.3 & 7.3 & 7.3 & 4.2 & 3.0 & 6.0 \\
u24g & 5.2 & 5.7 & 8.3 & 8.2 & 8.8 & 5.0 & 3.4 & 6.9 \\
u32g & 5.5 & 6.4 & 9.9 & 9.0 & 9.6 & 5.4 & 3.8 & 7.7 \\
u48g & 5.9 & 7.5 & 11.4 & 10.0 & 11.3 & 6.5 & 4.2 & 8.8 \\
u96g & 7.2 & 10.0 & 15.7 & 11.7 & 13.6 & 7.3 & 4.8 & 11.2 \\
\hline
\end{tabular}

\subsection{Results of dSTEC-GPS assessment}

Previous detailed analysis was based on the assessment of VTEC-Jason over the oceans. Another complementary assessment was performed by dSTECGPS observation, typically over the continental regions and with higher accuracy (at the level of $0.01 \mathrm{TECU}$ ) of the reference carrier phase measurements, in order to study the differences in performance within the higher time resolution GIMs. The results are summarized in Table 7. Similar with tables of VTEC-Jason assessment (Table 4, Table 5, and Table 6), the dSTEC accuracy of GIMs with high temporal resolution (uqrg, u02g, u03g, u04g) can be regarded as consistent with the VTEC one, also for low latitudinal bands, taking into account Figure 3 and that the dSTEC study has been done during one year (2015) close to solar maximum. The GIM time resolution results when the VTEC error significantly starts to increase, vs. latitude, which have been detailed in such a tables, are summarized in Figure 10. It can be seen than a resolution of 30 minutes is able to provide equivalent results to 15 minutes in such a test, either in the most accurate test (GPS dSTEC) and under Solar Maximum conditions, but one hour (right below 90 minutes in our study) is fine at mid and high latitude.

\section{Conclusions}

By comparing with altimeter data from Jason over the oceans, we have performed a comprehensive study about the behaviour during 1.5 solar cycles of 12 GIMs at increasing 24-hour divisor time intervals, derived from the original one at 15 minutes (uqrg, computed by UPC-IonSAT), 30 minutes (u02g), 45 minutes (u03g), 1 hour (u04g), 1 hour 30 minutes (u06g), 2 hours (u08g), 3 hours $(\mathrm{u} 12 \mathrm{~g}), 4$ hours $(\mathrm{u} 16 \mathrm{~g}), 6$ hours $(\mathrm{u} 24 \mathrm{~g}), 8$ hours $(\mathrm{u} 32 \mathrm{~g}), 12$ hours $(\mathrm{u} 48 \mathrm{~g})$ and 24 hours $(\mathrm{u} 96 \mathrm{~g})$. We have found that the standard deviation of the difference 
Table 7 RMS of dSTEC-GPS assessment in TECUs with 59 global distributed receivers during four days in 2015: 082, 146, 280, 330, in TECUs. The values showing a first increase of 0.1 TECU in the RMS in each given latitudinal band are represented in bold.

\begin{tabular}{ccccccccc}
\hline GIM & $-60^{\circ}$ & $-40^{\circ}$ & $-20^{\circ}$ & $0^{\circ}$ & $20^{\circ}$ & $40^{\circ}$ & $60^{\circ}$ & Overall \\
\hline uqrg & 3.00 & 3.60 & 5.69 & 6.91 & 5.61 & 2.42 & 2.16 & 4.25 \\
u02g & 2.97 & 3.59 & 5.71 & 6.88 & 5.62 & 2.42 & 2.15 & 4.25 \\
u03g & 2.96 & 3.60 & 5.76 & $\mathbf{7 . 0 0}$ & 5.67 & 2.45 & 2.16 & 4.29 \\
u04g & 2.98 & 3.66 & $\mathbf{5 . 9 0}$ & 7.03 & $\mathbf{5 . 7 6}$ & 2.49 & 2.19 & $\mathbf{4 . 3 6}$ \\
u06g & $\mathbf{3 . 0 9}$ & $\mathbf{3 . 8 0}$ & 6.25 & 7.47 & 6.14 & $\mathbf{2 . 6 7}$ & $\mathbf{2 . 2 5}$ & 4.62 \\
u08g & 3.24 & 4.07 & 6.83 & 7.81 & 6.60 & 2.90 & 2.38 & 4.96 \\
u12g & 3.73 & 4.88 & 8.07 & 9.10 & 7.97 & 3.51 & 2.57 & 5.89 \\
u16g & 4.22 & 5.67 & 9.08 & 10.23 & 9.23 & 4.11 & 2.80 & 6.73 \\
u24g & 4.70 & 6.68 & 10.65 & 12.68 & 11.22 & 5.16 & 3.20 & 8.14 \\
u32g & 5.48 & 8.26 & 12.23 & 14.85 & 13.37 & 5.97 & 3.70 & 9.56 \\
u48g & 6.57 & 10.18 & 13.72 & 15.66 & 14.59 & 6.46 & 3.91 & 10.47 \\
u96g & 7.77 & 11.25 & 18.75 & 18.97 & 17.30 & 7.44 & 4.49 & 12.80 \\
\hline
\end{tabular}

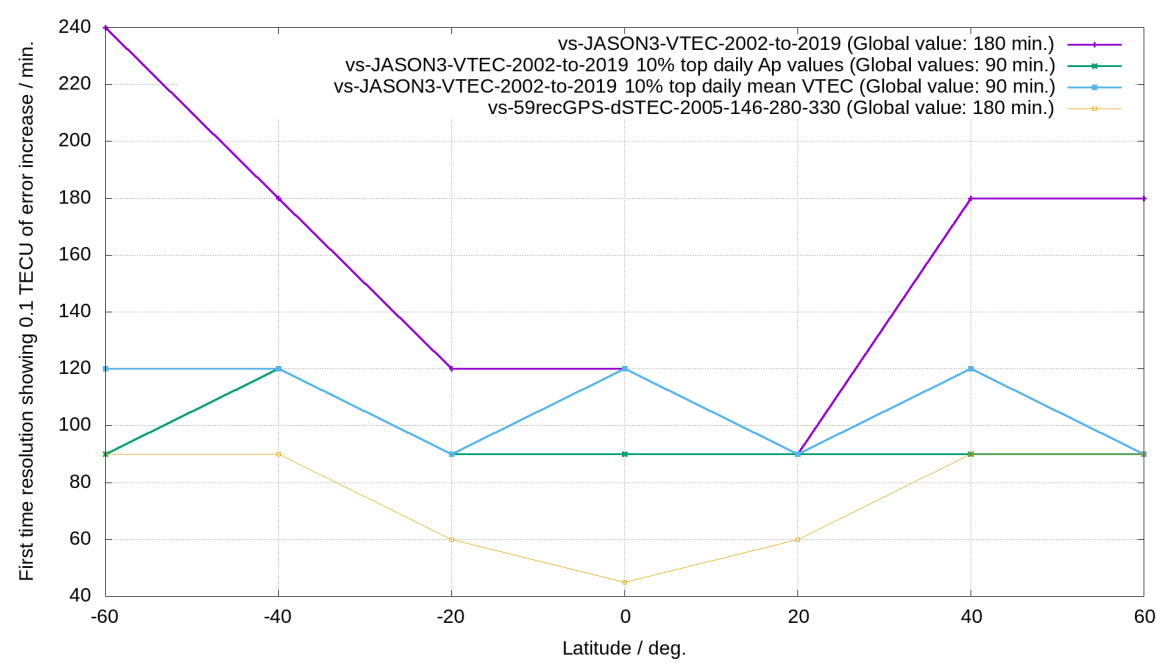

Fig. 10 GIM time resolution (in minutes) vs. latitude (degrees), when a first increase of 0.1 TECU of overall GIM VTEC error is detected, vs. JASON VTEC altimeters (standard deviation since day 26, 2002 up to day 335, 2019), only considering the top $10 \%$ of highest mean VTEC, and only the top $10 \%$ of highest daily Ap value, and finally vs. GPS dSTEC measurements, during days 82, 146, 280 and 330 of 2015.

between VTEC-GIM and VTEC-Jason is consistent with the evolution of solar cycle for these 12 different GIMs and is subject to the variation of geomagnetic activity when the solar activity is high. The variation of standard deviation appears to be intense under high geomagnetic activity. The accuracy of GIM is mainly affected by both solar and geomagnetic activity. Furthermore, the behaviour of standard deviation is diverse at different latitudes. The difference between GIMs with various temporal resolutions become more noticeable at equator (around $0^{\circ}$ degree) and low latitudinal bands (around $\pm 20^{\circ}$ degree). In addition, the accuracy of GIM at northern hemisphere is higher than at 
southern hemisphere due to the distribution of GNSS receivers.

As it has been detailed in the figures and tables of previous section, the discrepancy between GIMs of different temporal resolutions is obvious and should not be ignored. Considering the influence of solar-geomagnetic activity and the behaviour of standard deviation from VTEC-Jason assessment as well as RMS from dSTEC-GPS assessment at different latitudes, we have shown that a time resolution of 1 hour or higher (uqrg, u02g, u03g and u04g GIMs in this study) keep the accuracy of high-rate GIM (15 min.) at 0.1 TECU level. This recommended time resolution for GIMs is significantly lower that the standard time resolution in IGS GIMs (2 hours). We suggest to the corresponding scientific community to take this conclusion into account.

\section{Acknowledgements}

The first author is grateful to the financial support of China Scholarship Council(CSC). The Group for Jason-altimeter data were obtained from the NASA EOSDIS Physical Oceanography Distributed Active Archive Center (PO.DAAC) at the Jet Propulsion Laboratory, Pasadena, CA (http://dx . doi.org/10.5067/GHGMR-4FJ01) and the National Oceanic and Atmospheric Administration(NOAA). And we are also thankful to GFZ and SWPC for providing Ap index and F10.7 index, respectively. Also many thanks to Heng Yang for his kind advice of GIM id.

\section{References}

1. Azpilicueta, F., Brunini, C.: Analysis of the bias between TOPEX and GPS vTEC determinations. Journal of Geodesy 83(2), 121-127 (2009)

2. Bidaine, B., Lonchay, M., Warnant, R.: Galileo single frequency ionospheric correction: performances in terms of position. GPS solutions 17(1), 63-73 (2013)

3. Dach, R., Jean, Y.: Igs technical report 2012 (2013)

4. Feltens, J.: The activities of the ionosphere working group of the International GPS Service (IGS). GPS solutions 7(1), 41-46 (2003)

5. Goss, A., Schmidt, M., Erdogan, E., Görres, B., Seitz, F.: High-resolution vertical total electron content maps based on multi-scale b-spline representations. In: Annales Geophysicae, vol. 37 (2019)

6. Gurevich, A.: Nonlinear phenomena in the ionosphere, vol. 10. Springer Science \& Business Media (2012)

7. Hernández-Pajares, M., Juan, J., Sanz, J.: Neural network modeling of the ionospheric electron content at global scale using GPS data. Radio Science 32(3), 1081-1089 (1997)

8. Hernández-Pajares, M., Juan, J., Sanz, J.: New approaches in global ionospheric determination using ground GPS data. Journal of Atmospheric and Solar-Terrestrial Physics 61(16), 1237-1247 (1999)

9. Hernández-Pajares, M., Juan, J., Sanz, J.: Medium-scale traveling ionospheric disturbances affecting gps measurements: Spatial and temporal analysis. Journal of Geophysical Research: Space Physics 111(A7) (2006)

10. Hernández-Pajares, M., Juan, J., Sanz, J., Colombo, O.L.: Application of ionospheric tomography to real-time GPS carrier-phase ambiguities Resolution, at scales of 400 $1000 \mathrm{~km}$ and with high geomagnetic activity. Geophysical Research Letters 27(13), 2009-2012 (2000) 
11. Hernández-Pajares, M., Juan, J., Sanz, J., Orus, R., García-Rigo, A., Feltens, J., Komjathy, A., Schaer, S., Krankowski, A.: The IGS VTEC maps: a reliable source of ionospheric information since 1998. Journal of Geodesy 83(3-4), 263-275 (2009)

12. Hernández-Pajares, M., Lyu, H., Garcia-Fernandez, M., Orus-Perez, R.: A new way of improving global ionospheric maps by ionospheric tomography: consistent combination of multi-gnss and multi-space geodetic dual-frequency measurements gathered from vessel-, leo-and ground-based receivers. Journal of Geodesy 94(8), 1-16 (2020)

13. Hernández-Pajares, M., Roma-Dollase, D., Krankowski, A., García-Rigo, A., OrúsPérez, R.: Methodology and consistency of slant and vertical assessments for ionospheric electron content models. Journal of Geodesy (doi:10.1007/s00190-017-1032-z), $1-10(2017)$

14. Imel, D.A.: Evaluation of the topex/poseidon dual-frequency ionosphere correction. Journal of Geophysical Research: Oceans 99(C12), 24,895-24,906 (1994)

15. Jee, G., Lee, H.B., Kim, Y., Chung, J.K., Cho, J.: Assessment of gps global ionosphere maps (gim) by comparison between code gim and topex/jason tec data: Ionospheric perspective. Journal of Geophysical Research: Space Physics 115(A10) (2010)

16. Kivelson, A.: Introduction to space physics. Cambridge university press (1995)

17. Klobuchar, J.A.: Ionospheric time-delay algorithm for single-frequency gps users. IEEE Transactions on aerospace and electronic systems (3), 325-331 (1987)

18. Komjathy, A.: Global ionospheric total electron content mapping using the global positioning system. Ph.D. thesis, University of New Brunswick Fredericton (1997)

19. Laundal, K.M., Cnossen, I., Milan, S.E., Haaland, S., Coxon, J., Pedatella, N., Förster M., Reistad, J.P.: North-south asymmetries in earths magnetic field. Space Science Reviews 206(1-4), 225-257 (2017)

20. Li, M., Yuan, Y., Wang, N., Li, Z., Huo, X.: Performance of various predicted gnss global ionospheric maps relative to gps and jason tec data. GPS Solutions 22(2), 55 (2018)

21. Orús, R., Hernández-Pajares, M., Juan, J., Sanz, J.: Improvement of global ionospheric VTEC maps by using kriging interpolation technique. Journal of Atmospheric and Solar-Terrestrial Physics 67(16), 1598-1609 (2005)

22. Roma-Dollase, D., Hernández-Pajares, M., Krankowski, A., Kotulak, K., GhoddousiFard, R., Yuan, Y., Li, Z., Zhang, H., Shi, C., Wang, C., et al.: Consistency of seven different gnss global ionospheric mapping techniques during one solar cycle. Journal of Geodesy 92(6), 691-706 (2018)

23. Schaer, S.: Mapping and predicting the earths ionosphere using the Global Positioning System. 1999. 205p. Ph.D. thesis, Ph. D. dissertation. University of Bern, Bern, Switzerland (1999)

24. Schaer, S., Gurtner, W., Feltens, J.: IONEX: The ionosphere map exchange format version 1. In: Proceedings of the IGS AC workshop, Darmstadt, Germany, vol. 9 (ftp://igs.org/pub/data/format/ionex1.pdf) (1998)

25. Yuan, Y., Wang, N., Li, Z., Huo, X.: The beidou global broadcast ionospheric delay correction model (bdgim) and its preliminary performance evaluation results. Navigation 66(1), 55-69 (2019)

26. Zhang, Q., Zhao, Q.: Analysis of the data processing strategies of spherical harmonic expansion model on global ionosphere mapping for moderate solar activity. Advances in Space Research 63(3), 1214-1226 (2019)

27. Zhao, B., Wang, M., Yu, T., Wan, W., Lei, J., Liu, L., Ning, B.: Is an unusual large enhancement of ionospheric electron density linked with the 2008 great wenchuan earthquake? Journal of Geophysical Research: Space Physics 113(A11) (2008)

\section{Author contributions}

QL performed the research, MHP and QL designed the research and wrote the paper, and HL and AG helped to refine the ideas and to write the paper. 


\section{Data Availability Statement}

The UQRG GIMs, baseline of this work, are openly accessible from IGS server at ftp://cddis.gsfc.nasa.gov/gps/products/ionex/YEAR/DOY/uqrgDOYO. YYi.Z, being YEAR and YY the 4- and 2- digits year identifiers, and DOY is the day of year, all of them with leading zeroes when needed (see for instance ftp://cddis.gsfc.nasa.gov/gps/products/ionex/2002/003/uqrg0030.02i. $\mathrm{Z})$. Any missing file can be requested from the authors, in particular from Manuel Hernández-Pajares (manuel.hernandez@upc.edu). 